\title{
Right ventricular undersizing is associated with increased 1-year mortality
}

\author{
Masashi Kawabori, MD, ${ }^{\mathrm{a}}$ Andre C. Critsinelis, MD, ${ }^{\mathrm{b}}$ Camille E. Hironaka, BS, ${ }^{\mathrm{a}}$ \\ Frederick Y. Chen, MD, PhD, ${ }^{a}$ Yong Zhan, MD, ${ }^{a}$ Katherine L. Thayer, MPH, ${ }^{\mathrm{c}}$ and Gregory S. Couper, $\mathrm{MD}^{\mathrm{a}}$
}

\section{ABSTRACT}

Objectives: Right heart hemodynamic management is critical, because many postheart transplantation ( $H T x$ ) complications are related to right ventricular (RV) failure. However, current guidelines on size and sex matching rely primarily on weight matching, with recent literature using total ventricular mass (TVM), which places less emphasis on the impact of RV mass (RVM) matching. The aim of the present study was to analyze the relationship of RVM matching and survival after HTX.

Methods: We performed the retrospective analysis using the UNOS database of adult HTx performed between January 1997 and December 2017. Previously validated equations were used to calculate TVM and RVM. The percent difference in ventricular mass in the donor and recipient pair was used for the size mismatch. All donor-recipient pairs were divided into 4 RVM groups by their mismatch ratio. We analyzed RVM matching and explored how RVM undersizing impacted outcomes. The primary outcome measure was 1-year survival; secondary outcomes measured included stroke and dialysis within 1 year and functional status.

Results: A total of 38,740 donor-recipient pairs were included in our study. The 4 RVM match groupings were as follows: $<0 \%, 0 \%$ to $20 \%, 20 \%$ to $40 \%$, and $>40 \%$. Utilization of donors who were older and of female sex resulted in greater RVM undersizing. Survival analysis demonstrated patients with RVM undersizing had worse 1-year survival $(P<.001)$. RVM undersizing was an independent predictor of higher 1-year mortality (hazard ratio, 1.23; 95\% confidence interval, 1.11 to 1.34 ; $P<.001$ ). RVM undersizing was also associated with higher rates of dialysis within 1 -year of transplantation and poorer postoperative functional status.

Conclusions: RVM undersizing is an independent predictor for worse 1-year survival. Donors who are older and female have lower absolute predicted RVM and may be predisposed to RVM undersizing. RVM-undersized transplantation requires careful risk/benefit considerations. (J Thorac Cardiovasc Surg 2021;161:1048-59)

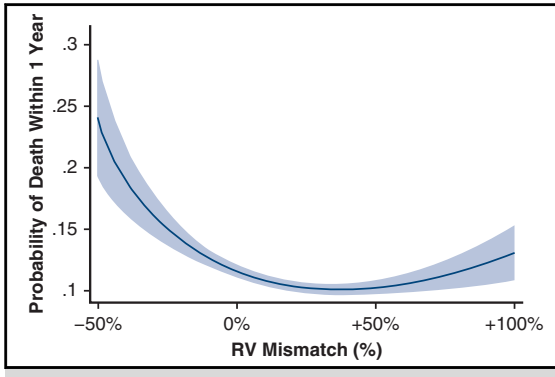

The association between right ventricular mass matching and 1-year mortality.

CENTRAL MESSAGE

Right ventricular mass undersiz-

ing was a predictor of worse

1-year survival after heart trans-

plantation. Older or female

donors have lower right ventric-

ular mass, which may predispose

to undersizing.

\section{PERSPECTIVE}

Current guidelines use only weight for donor recipient heart size matching, which limits prognostication. Right ventricular mass (RVM) matching takes age, sex, height, and weight into consideration. RVM undersizing is predictor of worse 1-year survival. Further research on RVM may lead to improved donor heart selection and post-transplantation survival and outcomes.

See Commentaries on pages 1060, 1061, and 1062.
Donor and recipient heart size match is critical in adult heart transplantation. Right ventricular mass undersizing was associated with worse 1-year survival, higher rate of dialysis within 1 year, and poorer postoperative functional status. Donors who are older and female have lower right

From the a Division of Cardiac Surgery, CardioVascular Center, Tufts Medical Center, Boston, Mass; ${ }^{\mathrm{b}}$ Department of Surgery, Mount Sinai Medical Center, Miami Beach, Fla; and ${ }^{\mathrm{c}}$ Division of Cardiology, CardioVascular Center, Tufts Medical Center, Boston, Mass.

Read at the 100th Annual Meeting of The American Association for Thoracic Surgery: A Virtual Learning Experience, May 22-23, 2020.

Received for publication June 12, 2020; revisions received Nov 4, 2020; accepted for publication Nov 6, 2020; available ahead of print Dec 11, 2020. ventricular mass and may predispose to right ventricular mass undersizing.

Current International Society of Heart and Lung Transplantation (ISHLT) guideline recommendations primarily use weight for donor-recipient heart size matching. ${ }^{1}$ This

Address for reprints: Masashi Kawabori, MD, 800 Washington St, South Building, 6th Floor, Boston, MA 02111 (E-mail: kawabori.masashi@gmail.com). 0022-5223

Copyright (C) 2020 The Author(s). Published by Elsevier Inc. on behalf of The American Association for Thoracic Surgery. This is an open access article under the CC BY-NC-ND license (http://creativecommons.org/licenses/by-nc-nd/4.0/). https://doi.org/10.1016/j.jtcvs.2020.11.156 


\section{Abbreviations and Acronyms \\ HTx $=$ heart transplantation \\ ISHLT $=$ International Society of Heart and Lung Transplantation \\ LVAD $=$ left ventricular assist device \\ LVM = left ventricular mass \\ $\mathrm{pHM}=$ predicted heart mass \\ $\mathrm{RV} \quad=$ right ventricular \\ $\mathrm{RVM}=$ right ventricular mass \\ $\mathrm{TVM}=$ total ventricular mass \\ UNOS $=$ United Network for Organ Sharing}

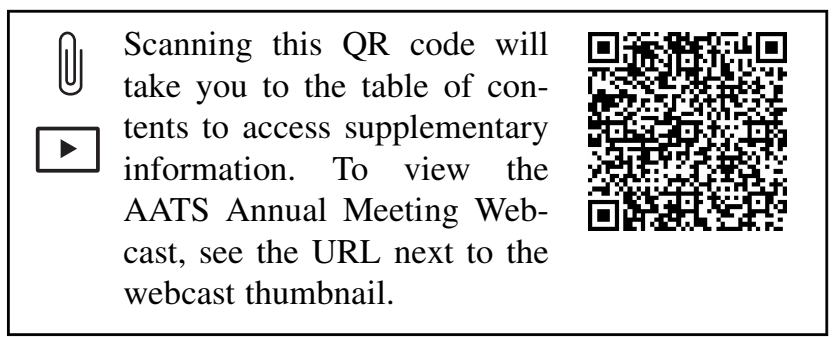

methodology can be a poor predictor of outcomes, however, particularly in male recipients of female donor hearts, because cardiac mass can vary by sex. ${ }^{2,3}$ Previous studies have suggested the utility of size matching using a more comprehensive calculation for predicted heart mass $(\mathrm{pHM})$ that incorporates height, weight, age, and sex. ${ }^{4,5}$ Recent studies using these additional variables have shown better prognostication of heart transplantation (HTx) survival compared with traditional weight-based methodology. ${ }^{6-9}$

Right heart hemodynamic management is critical after HTx. The use of size-matching calculators that incorporate only total ventricular mass (TVM) can be biased by the left ventricular mass (LVM), because LVM is significantly larger than right ventricular mass (RVM). Therefore, using TVM-based equations may underemphasize the impact of RVM matching.

Validated equations for estimated RVM and LVM have been published previously. ${ }^{4,5,10-12}$ RVM calculation uses age as a variable, whereas LVM calculation does not. This may be of interest because donor and recipient age discrepancy has been associated with adult HTx mortality. ${ }^{8}$ Because LVM dominates the bulk of TVM, with less impact from RVM, TVM calculators may underestimate the impact of donor and recipient age on outcomes. We hypothesize that a RVM calculator may predict HTx outcomes more accurately by more properly weighting RVM and age matching.

To our knowledge, no previous studies have examined the effect of RVM matching on HTx outcomes. The primary aim of the present study was to analyze the impact of RVM matching in adult HTx on 1-year survival. We also sought to compare survival prediction of RVM mismatching to TVM mismatching and to analyze the outcomes of current guideline recommendations.

\section{METHODS}

\section{Data Source}

We used the United Network for Organ Sharing (UNOS) Database as a source of deidentified donor and recipient HTx data. This database consists of prospectively collected donor and recipient demographic and transplantation data for all organ transplantations performed in the United States. Institutional Review Board approval was obtained.

\section{Inclusion and Exclusion Criteria}

We included adult HTx recipients who underwent transplantation between January 1997 and December 2017. There were 49,819 HTx donorrecipient pairs in this time period. We used similar exclusion criteria to those used in previous studies. ${ }^{9}$ We excluded patients with previous HTx and combined multiple organ transplantation. We also excluded recipients age $<18$ years or $>70$ years, with height $<140 \mathrm{~cm}$ or $>210 \mathrm{~cm}$, weight less than $40 \mathrm{~kg}$ or greater than $130 \mathrm{~kg}$, body mass index (BMI) $<15 \mathrm{~kg} / \mathrm{m}^{2}$ or $>40 \mathrm{~kg} / \mathrm{m}^{2}$, and serum creatinine $>5 \mathrm{mg} / \mathrm{dL}$ or blood urea nitrogen $>100 \mathrm{mg} / \mathrm{dL}$. We also limited donor-recipient weight match from $-75 \%$ to $+100 \%$ and RV or LV mismatch from $-50 \%$ to $+100 \%$. Finally, patients with insufficient data provided for calculating estimated RVM or LVM were excluded. After exclusions, 38,740 donor-recipient pairs were included (Figure E1).

\section{Ventricular Mass Calculation}

Estimated ventricular mass was calculated for donors and recipients using equations derived from the Multi-Ethnic Study of Atherosclerosis (MESA) by Bluemke and colleagues. ${ }^{4,10-12}$ The equations are as follows:

Predicted LVM $=\alpha \times$ Height $^{0.54}($ meters $) \times$ Weight $^{0.61}($ kilograms $)$,

where $\alpha=6.82$ for women and 8.25 for men,

and

Predicted RVM $=\alpha \times$ Age $^{-0.32}($ years $) \times$ Height $^{1.135}($ meters $) \times$ Weight $^{0.315}$ (kilograms)

where $\alpha=10.59$ for women and 11.25 for men. ${ }^{9}$

Predicted TVM was calculated by adding predicted RVM and predicted LVM. Whereas the equation for RVM uses age as a factor, the equation for predicted LVM does not.

\section{Donor-Recipient Cardiac Mass Matching Formula}

We used the following equation to calculate mismatch percentage:

Predicted Cardiac Mass Mismatch ( $\%)=($ Donor Mass - Recipient Mass $) /$ Recipient Mass $\times 100$

Negative values indicate undersizing. TVM and RVM mismatch was calculated using the donor and recipient TVM and RVM values.

\section{Statistical Analysis}

Baseline recipient characteristics are presented as median (interquartile range [IQR]) for continuous variables and as count ( $\%$ ) for categorical variables. RVM mismatch grouping was set up to allow for approximately even group size while also differentiating between undersizing and oversizing. The RVM mismatched groups were as follows: $<0 \%, 0 \%$ to $20 \%$, $20 \%$ to $40 \%$, and $\geq 40 \%$, with negative values indicating RV undersizing. 
A similar method was used for LVM mismatching with the aim of producing approximately even group sizes. The TVM grouping was as follows: $<-20 \%,-20 \%$ to $0 \%, 0 \%$ to $20 \%$, and $\geq 20 \%$. Kernel density plots were used to visually demonstrate the distribution of variables, and the Kolmogorov-Smirnov test was used to compare the distributions. A fractional polynomial regression prediction plot was calculated for the probability of death by 1 year after transplantation. Kaplan-Meier survival analysis was used to compare 1-year survival rates between groups, and the log-rank test was used to calculate $P$ values. Subanalysis of the impact of varying degrees of RVM undersizing was performed using KaplanMeier and log-rank tests. A multivariable Cox proportional hazards model was used to identify independent predictors of death within 1 year of transplantation. Variables included in our final proportional hazards model were selected based on evidence from the literature identifying important clinical factors that strongly impact survival after transplantation that were available in the dataset. The variance inflation factor (VIF) was calculated as a measure of collinearity between variables in our final model. In a stepwise fashion, variables with VIF $>10$ were removed from the model (one at a time beginning the variables with the higher VIF) until there were no remaining variables with VIF $>10$. The final model had a Harrell's C-index of 0.60. Proportional hazards assumptions were confirmed using Schoenfeld's residuals, and the linearity of continuous variables was confirmed using Martingale residuals.

The Shapiro-Wilk normality test was performed on each continuous variable. The $t$ test and analysis of variance were performed on normally distributed variables as appropriate, and the Mann-Whitney $U$ test was used for non-normally distributed variables. A $P$ value $<.05$ was considered statistically significant. All statistical analyses were performed with Stata 14.0 software (StataCorp, College Station, TX).

\section{RESULTS}

\section{Donor-Recipient Matching}

Figure 1 shows the distribution of RVM and TVM mismatch in all donor-recipient pairs; these distributions were significantly different from each other $(P<.001)$. The median RVM was $19.2 \%$ (IQR, $4.6 \%$ to $35.0 \%$ ), and median TVM was $0.5 \%$ (IQR, $-9.2 \%$ to $+12.9 \%$ ). RVM matching distribution had higher proportion of oversized patients and a wider distribution when compared with TVM matching. RVM was separated in 4 groups; patients with RVM undersizing were in one group $(<0 \% \mathrm{RVM}$ mismatch) and comprised $17.9 \%$ of the entire cohort $(\mathrm{n}=6920)$. TVM was separated into 4 groups; patients with TVM undersizing were spread across 2 groups $(<-20 \%$ and $-20 \%$ to $0 \%)$ with $7.9 \%(\mathrm{n}=2940)$ of the cohort in the $<-20 \%$ group and $41.0 \%(\mathrm{n}=15,888)$ in the $-20 \%$ to $0 \%$ group.

Figure E2 shows the distribution of donor-recipient sex match in the entire cohort. A total of 26.6\% of transplants had a sex mismatch. Female donor to male recipient pairings composed $15.7 \%$ of transplants $(\mathrm{n}=6071)$.

\section{Recipient Preoperative Characteristics}

Table 1 shows the recipient characteristics and hemodynamics by RVM mismatch groups. $P$ values for this table were calculated comparing undersized $(<0 \%)$ to oversized $(\geq 0 \%)$ groups. The RVM undersized group contained recipients with younger age, higher proportion of male recipients, higher proportion of female donors to male recipients, lower proportion of male donors to female recipients, and lower recipient pulmonary vascular resistance $(P<.001)$. All groups had between roughly $10.5 \%$ to $12.5 \%$ of recipients with a preoperative left ventricular assist device (LVAD) with no increase or decrease trend, despite a significant $P$ value of .006 . The age difference between recipient and donor was

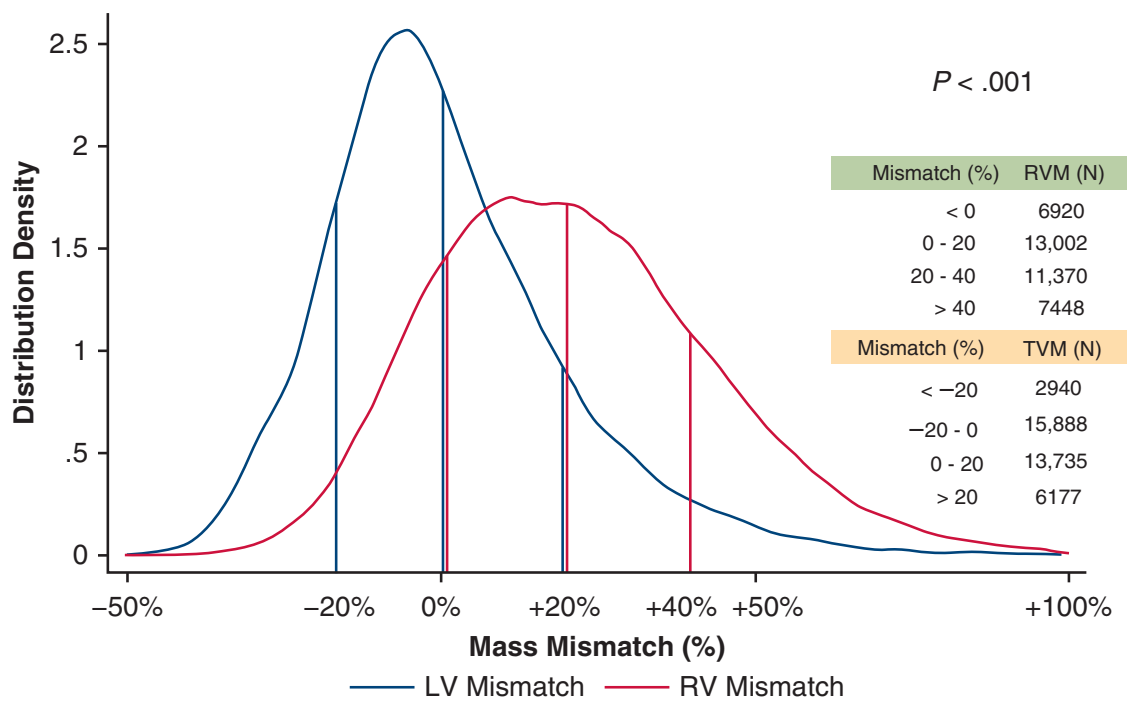

FIGURE 1. Distribution of donor-recipient mismatch for right ventricular mass (RVM; green) and total ventricular mass (TVM; yellow) for the heart transplantations included in the study. The 2 distributions differed significantly $(P<.001)$. Mass mismatch was subdivided into 4 groups for each metric; RVM cutoffs were $<0 \%, 0 \%$ to $+20 \%,+20 \%$ to $+40 \%$, and $>+40 \%$; TVM cutoffs were $<-20 \%,-20 \%$ to $0 \%, 0 \%$ to $+20 \%$, and $>+20 \%$. These groupings are delineated within the distribution curve. $L V$, Left ventricle; $R V$, right ventricle. 
TABLE 1. Preoperative patient characteristics by right ventricular mismatch subgroup

\begin{tabular}{|c|c|c|c|c|c|c|}
\hline \multirow[b]{2}{*}{ Characteristic } & \multirow[b]{2}{*}{$\begin{array}{l}\text { All patients } \\
(\mathbf{N}=38,740)\end{array}$} & \multicolumn{4}{|c|}{ Right ventricular mismatch subgroup } & \multirow[b]{2}{*}{$P$ value } \\
\hline & & $\begin{array}{c}<0 \% \\
(\mathbf{N}=6 \mathbf{6 9 2 0})\end{array}$ & $\begin{array}{l}\mathbf{0} \% \text { to }+\mathbf{2 0} \% \\
(\mathbf{N}=\mathbf{1 3 , 0 0 2})\end{array}$ & $\begin{array}{c}+20 \% \text { to }+\mathbf{4 0} \% \\
(\mathbf{N}=\mathbf{1 1 , 3 7 0 )}\end{array}$ & $>40 \%(N=7448)$ & \\
\hline \multicolumn{7}{|l|}{ Demographics } \\
\hline Recipient age, y, n (\%) & $55(46-62)$ & $47(34-57)$ & $54(45-61)$ & $57(50-62)$ & $59(53-64)$ & $<.001$ \\
\hline Female recipient, $\mathrm{n}(\%)$ & $9564(24.7)$ & $1224(17.7)$ & $2787(21.4)$ & $2687(23.6)$ & $2866(38.5)$ & $<.001$ \\
\hline $\begin{array}{l}\text { Female donor to male recipient, } \\
\mathrm{n}(\%)\end{array}$ & $6071(15.7)$ & $2654(38.4)$ & $2262(17.4)$ & $939(8.3)$ & $216(2.9)$ & $<.001$ \\
\hline $\begin{array}{l}\text { Male donor to female recipient, } \\
\mathrm{n}(\%)\end{array}$ & $4224(10.9)$ & $169(2.4)$ & $746(5.7)$ & $1252(11.0)$ & $1375(12.1)$ & $<.001$ \\
\hline $\begin{array}{l}\text { Recipient-donor age } \\
\text { difference, y, median (IQR)* }\end{array}$ & $22(10-33)$ & $3(-5$ to 11$)$ & $16(8-24)$ & $29(20-36)$ & $37(31-43)$ & $<.001$ \\
\hline \multicolumn{7}{|l|}{ Medical history } \\
\hline Previous sternotomy, n (\%) & $4046(10.4)$ & $6158(89.0)$ & $11,681(89.8)$ & $10,259(90.2)$ & $6596(88.6)$ & .001 \\
\hline \multicolumn{7}{|l|}{$\begin{array}{l}\text { Preoperative laboratory tests, } \\
\text { median (IQR) }\end{array}$} \\
\hline Total bilirubin, $\mathrm{mg} / \mathrm{dL}$ & $0.8(0.5-1.2)$ & $0.8(0.5-1.3)$ & $0.8(0.5-1.2)$ & $0.8(0.5-1.2)$ & $0.8(0.5-1.2)$ & $<.001$ \\
\hline Creatinine, mg/dL & $1.2(1.0-1.5)$ & $1.2(0.9-1.5)$ & $1.2(1.0-1.5)$ & $1.2(1.0-1.5)$ & $1.2(1.0-1.5)$ & $<.001$ \\
\hline Albumin, g/dL & $3.8(3.3-4.2)$ & $3.8(3.3-4.2)$ & $3.8(3.3-4.2)$ & $3.7(3.3-4.1)$ & $3.7(3.3-4.1)$ & .52 \\
\hline \multicolumn{7}{|l|}{ Preoperative hemodynamics } \\
\hline $\begin{array}{l}\text { Cardiac output, L/min, median } \\
\text { (IQR) }\end{array}$ & $4.4(3.5-5.4)$ & $4.4(3.5-5.5)$ & $4.4(3.6-5.4)$ & $4.4(3.5-5.4)$ & $4.1(3.3-5.1)$ & $<.001$ \\
\hline PCWP, mm Hg, median (IQR) & $18(12-25)$ & $19(12-25)$ & $18(12-25)$ & $18(12-25)$ & $18(12-25)$ & $<.001$ \\
\hline $\begin{array}{l}\text { PVR, Woods units, median } \\
\text { (IQR) }\end{array}$ & $2.36 \pm 1.87$ & $2.26 \pm 1.88$ & $2.30 \pm 1.91$ & $2.37 \pm 1.88$ & $2.52 \pm 1.76$ & $<.001$ \\
\hline $\begin{array}{l}\text { Systolic PAP, mm Hg, median } \\
\text { (IQR) }\end{array}$ & $40(31-51)$ & $40(30-50)$ & $40(31-50)$ & $40(31-51)$ & $41(32-52)$ & .03 \\
\hline $\begin{array}{l}\text { Diastolic PAP, mm Hg, median } \\
\text { (IQR) }\end{array}$ & $20(14-26)$ & $20(14-27)$ & $19(14-25)$ & $20(14-25)$ & $20(14-26)$ & $<.001$ \\
\hline LVAD, n $(\%)$ & $4528(11.7)$ & $742(10.7)$ & $1631(12.5)$ & $1375(12.1)$ & $780(10.5)$ & .006 \\
\hline
\end{tabular}

$P$ values were calculated comparing right ventricular $(\mathrm{RV})$ undersizing (mismatch $<0 \%$ ) vs RV oversizing (mismatch $>0 \%$ ). IQR, Interquartile range; $P C W P$, pulmonary capillary wedge pressure; $P V R$, pulmonary vascular resistance; $P A P$, Pulmonary artery pressure; $L V A D$, left ventricular assist device. $*$ Recipient-donor age difference $=$ recipient age - donor age.

significantly lower in the RV undersized group compared with the oversized group.

Recipient median RVM and LVM were $23.6 \mathrm{~g}$ (IQR, 21.3-25.8 g) and $159.3 \mathrm{~g}$ (IQR, 135.5-177.9 g), respectively. Donor median RVM and LVM were $28.3 \mathrm{~g}$ (IQR, 24.7$31.8 \mathrm{~g}$ ) and $155.4 \mathrm{~g}$ (IQR, 134.1-173.0 g), respectively. The ratio of RVM to TVM for recipients was $13.2 \%$ for recipient hearts and $15.5 \%$ for donor heart. LVM comprises the bulk of TVM in both groups.

Figure E3 shows donor LVM and RVM stratified by sex and age. Compared with female donors, male donors had greater RVMs (29.1 g [IQR, 25.7-32.4 g] vs $25.5 \mathrm{~g}$ [IQR, 22.2-29.2 g]; $P<.001)$ and LVMs (161.1 g [IQR, 144.0$177.6 \mathrm{~g}$ ] vs $132.0 \mathrm{~g}$ [IQR, 113.0-152.4 g]; $P<.001)$ compared with female donors. Absolute RVM showed a gradual decrease with increasing donor age.

Older donor age groups and female sex demonstrated a trend toward more negative RVM mismatch. For example, $48.9 \%$ male donors and $75.2 \%$ of females in their $60 \mathrm{~s}$ were associated with RVM undersizing, compared with only $3.2 \%$ of male donors and $9.7 \%$ of female donors in their $20 \mathrm{~s}$.

\section{Postoperative Survival and Complications}

Table 2 shows the postoperative complication rates within 1 year of transplantation across the 4 RVM matching groups. Waitlist time and ischemic time for HTx were similar among the groups, whereas the RVM undersizing group had higher rates of dialysis and death and worse functional status as measured by the Karnofsky Performance Status compared with oversized groups. Survival difference among the 4 groups were apparent within the first 90 days after transplantation.

Figure 2, A, shows the 1-year Kaplan-Meier survival curve for the 4 RVM matching groups. The undersized group had significantly worse survival compared with the other 3 groups, which showed similar outcomes ( $P$ not significant). This group had continuous separation 
from the other groups over the entire observation period. Figure 2, $B$, shows the 1-year Kaplan-Meier survival curve for the 4 TVM matching groups. The group with the greatest degree of TVM undersizing $(<-20 \%)$ had significantly worse survival compared with the other groups $(P<.001)$. The survival curves were not significantly different among the other groups $(P$ not significant). The most undersized group had an initial drop in the early postoperative period with a mild decline or parallel survival thereafter compared with the other groups.

Figure 3, A, shows the results of a 1-year survival probability analysis using the fractional polynomial regression for RVM mismatch. The $y$-axis demonstrates the probability of mortality within 1 year after transplantation. RVM mismatch showed a nadir at $+37.0 \%$. Recipients with RVM undersizing had a higher mortality rate compared with TVM- undersized recipients. Figure 3, $B$, shows the results of a 1-year survival probability analysis using the fractional polynomial regression for TVM. Figure 3, C, compares the 1-year survival probability analysis results among the RVM, TVM, and LVM mismatch groups. Compared with LVM and TVM, RVM was associated with the lowest mortality at higher (more positive) mismatch levels. The TVM curve shows a steeper elevation when undersized $(<0 \%)$ compared with RVM matching.

Table 3 shows the multivariable Cox proportional hazard analysis, independent predictors for 1-year mortality. The final model was adjusted for age, serum creatinine, ischemic time, pulmonary vascular resistance (PVR), and pretransplantation LVAD. In our multivariable model, RVM undersizing $(<0 \%)$ was an independent predictor for 1-year mortality (hazard ratio [HR], 1.22; 95\% confidence interval $[\mathrm{CI}], 1.11-1.34 ; P<.001)$. Although TVM undersizing $<-20 \%$ and female donor to male recipient pairing significantly impacted survival on univariable analysis, they were not independent predictors of mortality in our multivariable analysis (HR, 1.11; 95\% CI, 0.96-1.29; $P=.16$ and HR, $1.03 ; 95 \%$ CI, 0.92-1.15; $P=.58$, respectively).

\section{Projection of Current Guidelines}

Figure 4 shows the 1-year survival probability analysis using the fractional polynomial regression for weight mismatch comparing female donor to male recipient transplantations to all other transplantations. The non-female donor to male recipient group (red curve) shows primarily a flat curve with probability of death $<0.12$, with the exception of the left end of the curve, which shows a rapid increase in mortality risk. The 1-year mortality of this curve for $-30 \%$ of weight match is $11.0 \%$. The blue curve representing female donor to male recipient transplantations has a "bottom-rounded V shape" within the narrow range of weight matching, which has a probability of death $<0.12$ in an oversized donor. The 1-year mortality of this curve for $-20 \%$ weight match is $14.4 \%$. The blue curve showed a higher risk in all matching ranges compared with the red curve.

\section{DISCUSSION}

Appropriate donor-recipient heart matching is essential for optimizing HTx outcomes. Our study retrospectively analyzed 20 years of UNOS database transplantation outcomes for RVM matching, TVM matching, and current guideline recommendations using weight matching for donor-recipient cardiac size matching. Our study demonstrates that RVM comprises only a small portion $(13 \%$ $15 \%$ ) of predicted TVM, although clinically, management of RV hemodynamics is critical for optimum outcomes after HTx. Our primary finding was that patients with undersized RVM had worse survival on Kaplan-Meier survival analysis, with RVM undersizing identified as an independent

TABLE 2. Complications in the right ventricular mass matching subgroups

\begin{tabular}{|c|c|c|c|c|c|}
\hline \multirow[b]{2}{*}{ Complication } & \multicolumn{4}{|c|}{ Right ventricular mismatch subgroup } & \multirow[b]{2}{*}{$P$ value } \\
\hline & $\begin{array}{c}<<\% \\
(\mathbf{N}=\mathbf{6 9 2 0})\end{array}$ & $\begin{array}{l}0 \% \text { to }+20 \% \\
(N=13,002)\end{array}$ & $\begin{array}{c}+20 \% \text { to }+\mathbf{4 0} \% \\
(\mathbf{N}=\mathbf{1 1 , 3 7 0 )}\end{array}$ & $\begin{array}{c}>40 \% \\
(N=7448)\end{array}$ & \\
\hline Waitlist time, d, median (IQR) & $92(29-255)$ & $102(31-276)$ & $99(30-269)$ & $84(25-242.5)$ & .04 \\
\hline $\begin{array}{l}\text { Ischemic time, h, median } \\
\text { (IQR) }\end{array}$ & $3.2(2.4-3.8)$ & $3.1(2.4-3.8)$ & $3.1(2.4-3.8)$ & $3.1(2.3-3.8)$ & $<.001$ \\
\hline Stroke within 1 y, n (\%) & $158(2.3)$ & $316(2.4)$ & $281(2.5)$ & $205(2.8)$ & $<.001$ \\
\hline Dialysis within $1 \mathrm{y}, \mathrm{n}(\%)$ & $750(10.8)$ & $1308(10.1)$ & $1029(9.1)$ & $608(8.2)$ & $<.001$ \\
\hline Death within $90 \mathrm{~d}, \mathrm{n}(\%)$ & $572(8.3)$ & $958(7.4)$ & $808(7.1)$ & $543(7.3)$ & .004 \\
\hline Death within 1 y, n (\%) & $867(12.5)$ & $1400(10.8)$ & $1184(10.4)$ & $771(10.4)$ & $<.001$ \\
\hline KPS score, $\%$, median (IQR) & $80(50-100)$ & $90(60-100)$ & $90(60-100)$ & $90(60-100)$ & .003 \\
\hline
\end{tabular}

Postoperative complication rates (including stroke, dialysis, graft failure, and death) are reported as rate within 1 year of transplantation. Graft failure is defined as requirement for retransplantation. $P$ values were calculated comparing right ventricular (RV) undersizing (mismatch $<0 \%$ ) vs RV oversizing (mismatch $>0 \%$ ). IQR, Interquartile range; KPS, Karnofsky Performance Status. 


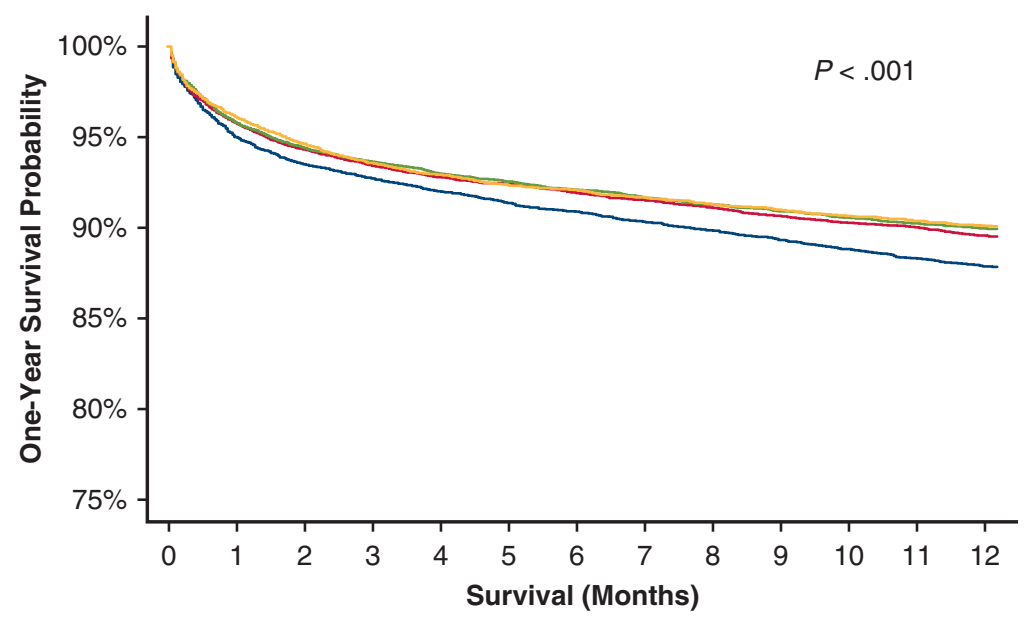

\begin{tabular}{|c|c|c|c|c|c|}
\hline Number at risk & & & & & \\
\hline$<0 \%$ & 6883 & 6349 & 6219 & 6103 & 5940 \\
\hline $0 \%$ to $+20 \%$ & 12,949 & 12,048 & 11,834 & 11,643 & 11,351 \\
\hline$+20 \%$ to $+40 \%$ & 11,320 & 10,565 & 10,385 & 10,236 & 10,003 \\
\hline$>+40 \%$ & 7405 & 6906 & 6787 & 6701 & 6559 \\
\hline
\end{tabular}

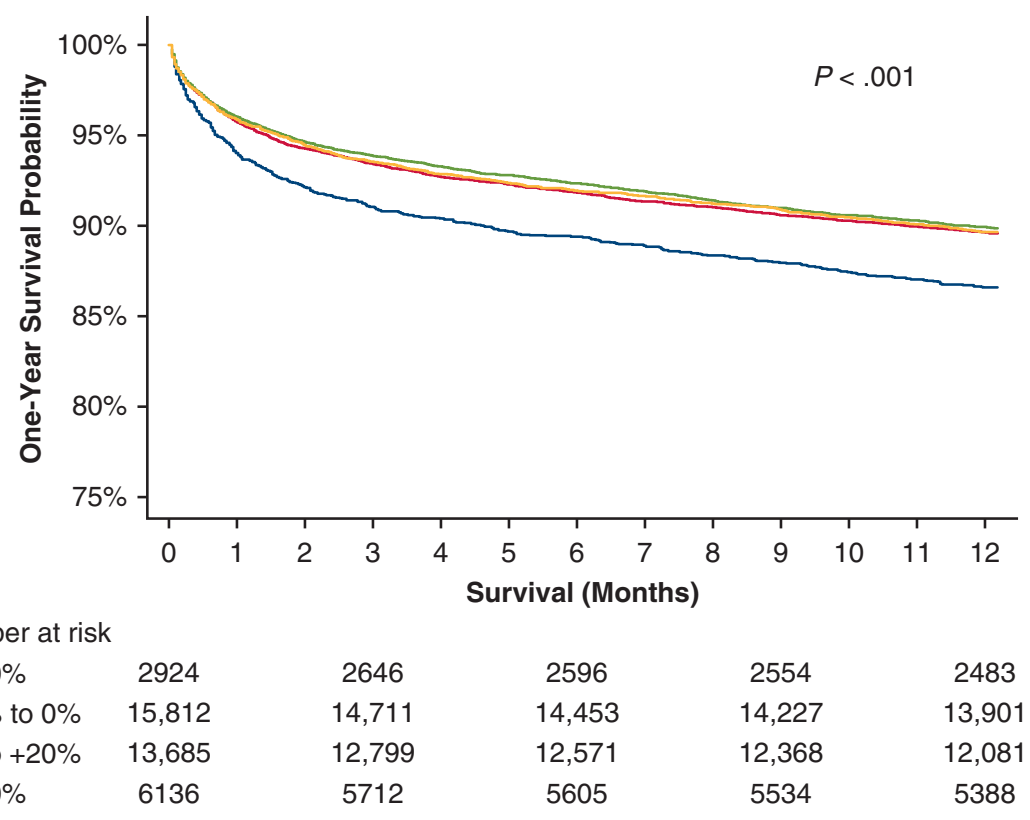

FIGURE 2. Kaplan-Meier curves demonstrating 1-year survival stratified by different right ventricular mass (RVM) mismatch groups and total ventricular mass (TVM) mismatch groups. A, RVM mismatch $<0 \%$ had significantly poorer survival compared with other RVM mismatch groups $(P<.001)$. B, TVM mismatch $<-20 \%$ had significantly poorer survival compared with other TVM mismatch groups. Of note, the group consisting of TVM $-20 \%$ to $0 \%$ mismatch did not have poorer survival compared with the TVM oversized groups $(0 \%$ to $+20 \%$ and $>+20 \%)$. Replicated images with $95 \%$ confidence intervals are shown in Figure E4.

risk factor for poorer 1-year survival after HTx. RVM undersizing was also associated with higher rates of postoperative dialysis and poorer postoperative functional status. In using the predicted ventricular mass calculations, females and older donors had lower absolute TVMs and
RVMs compared with males, which may predispose them to RVM undersizing.

With more than 5500 patients worldwide undergoing HTx each year, and given the plethora of clinical factors that should be taken into account, assessment of donor- 
recipient matching should be evaluated on a case-by-case basis. ${ }^{13}$ Careful consideration should be paid to such factors as sex, body size, age, urgency of HTx, and pulmonary vascular resistance. There are also institutional factors that vary among transplantation centers, such as geography for ischemic time, institutional experiences, case volumes, recent clinical outcomes, and aggressiveness for risktaking. Given the large number of continuous variables involved, it is not easy to define universal cutoff lines for donor recipient acceptance. However, it is our hope that in clarifying guidelines and important parameters, multidisciplinary heart teams can more accurately assess the riskbenefit ratios for their patients in developing management strategies and in selecting optimum donors.

Current ISHLT guidelines for donor-recipient matching using weight-based methodologies are supported by $\mathrm{C}$-level evidence. ${ }^{1}$ According to their guidelines, "the use of hearts from donors whose body weight is no greater than $30 \%$ below that of the recipient is uniformly safe. Furthermore, a male donor of average weight $(70 \mathrm{~kg})$ can be safely used for any size recipient irrespective of weight. Use of a female donor whose weight is more than $20 \%$ lower than that of a male recipient should be viewed with caution."1 Several studies have identified limitations of these guidelines, ${ }^{2-7}$ and Patel and colleagues ${ }^{2}$ found that donorrecipient weight ratio did not predict mortality after HTx, but identified recipients with elevated pulmonary vascular resistance who received undersized hearts as at greater risk of poorer survival. Russo and colleagues ${ }^{14}$ showed careful selection beyond clinical guidelines is prudent in recipients with high BMI. Their study identified recipient BMI $>35 \mathrm{~kg} / \mathrm{m}^{2}$ as associated with higher morbidity and mortality. ${ }^{14}$ Khush and colleagues ${ }^{3}$ reported a strong association between donor-recipient sex mismatch and reduced survival after HTx; male recipients of female allografts had a $10 \%$ higher adjusted mortality relative to male recipients of male allografts. ${ }^{3}$ Higher donor age also has been associated with poorer survival. ${ }^{15-17}$ In these analyses, it is clear that the intricacies of donor-recipient matching are significantly more complex than the current guidelines suggest.

Given the importance of right heart hemodynamic management after HTx, we sought to explore the impact of RVM donor-recipient matching. To estimate ventricular mass, we relied on a previously published and validated equations. ${ }^{4,5,9}$ Reed and colleagues ${ }^{9}$ used pHM to stratify the risk of mortality at 1 year due to donor recipient heart mass mismatching. They commented that sex mismatch could result in pHM mismatch and demonstrated that a $\mathrm{pHM}<-15 \%$ was associated with higher rates of acute rejection and mortality. Several other studies have highlighted TVM as being more clinically appropriate for size matching than weight matching. Kransdorf and colleagues ${ }^{7}$ showed an association between pHM undersizing (range, $0.54 \%-0.86 \%$ ) and increased mortality (HR, 1.34). The

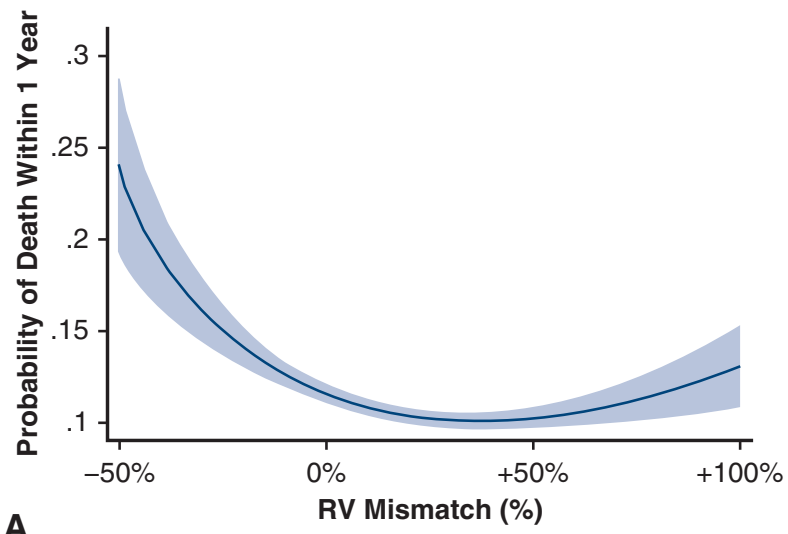

A
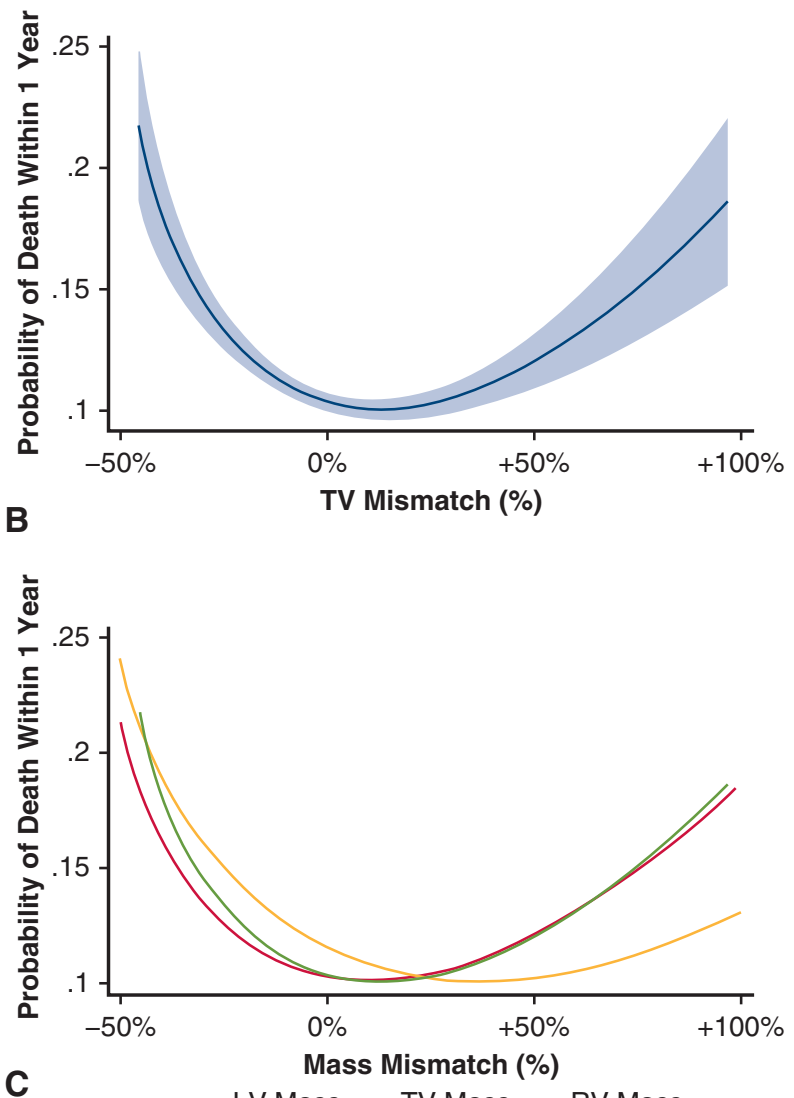

— LV Mass — TV Mass — RV Mass

FIGURE 3. Fractional polynomial regression models demonstrating the probability of death within 1-year of transplantation by right ventricular mass (RVM) mismatch (A) and total ventricular mass (TVM) mismatch (B). Blue shading indicates $95 \%$ confidence intervals. C, Comparison of RVM, TVM, and left ventricular (LVM) mass. For RVM, 6920 patients $(17.9 \%)$ had RVM undersizing $(<0 \%), 27,811$ patients $(71.8 \%)$ had RVM oversizing from $0 \%$ to $+50 \%$, and 4009 patients $(10.3 \%)$ had RVM oversizing from $+50 \%$ to $+100 \%$. For TVM, 18,828 patients $(48.6 \%)$ had TVM undersizing $(<0 \%), 19,208$ (49.6\%) had TVM oversizing from $0 \%$ to $+50 \%$, and $704(1.8 \%)$ had TVM oversizing from $+50 \%$ to $+100 \%$. $R V$, Right ventricle; $T V$, total ventricle; $L V$, left ventricle.

use of pHM could improve donor heart utilization and post-transplantation survival. ${ }^{7}$ For recipients with an 
TABLE 3. Multivariable Cox proportional hazard analysis identifying independent predictors of 1-year mortality

\begin{tabular}{llccc}
\hline \multicolumn{1}{c}{ Variable } & HR & SE & 95\% CI & P value \\
\hline Recipient age (y) & 1.01 & 0.002 & $1.006-1.12$ & $1.42-1.58$ \\
Serum creatinine (mg/dL) & 1.50 & 0.04 & $1.10-1.18$ & $<.001$ \\
\hline Ischemic time (h) & 1.14 & 0.02 & $1.02-1.05$ & $<.001$ \\
PVR (Woods units) & 1.03 & 0.01 & $1.01-1.25$ & .001 \\
Pretransplantation LVAD & 1.13 & 0.06 & $0.96-1.29$ & .03 \\
TVM undersize $<-20 \%$ & 1.11 & 0.09 & $1.11-1.34$ & $0.92-1.15$ \\
RVM undersize $<0 \%$ & 1.23 & 0.06 & .001 \\
Female donor to male & 1.03 & 0.06 & .001 \\
\hline
\end{tabular}

HR, Hazard ratio; $S E$, standard error; $C I$, confidence interval; $P V R$, pulmonary vascular resistance; $L V A D$, left ventricular assist device; TVM, total ventricular mass; $R V M$, right ventricular mass.

LVAD, donor oversizing with a BMI ratio $>1.2$ showed improved long-term graft survival. ${ }^{18}$ As our analysis demonstrated, older and female donors had smaller RVM, leading us to believe that RVM undersizing may be a contributing factor to these mismatch analysis findings. To our knowledge, there have been no studies examining RVM matching reported to date.

In our analysis of HTxs performed over a 20-year period in the United States, patients in the undersized group (RVM

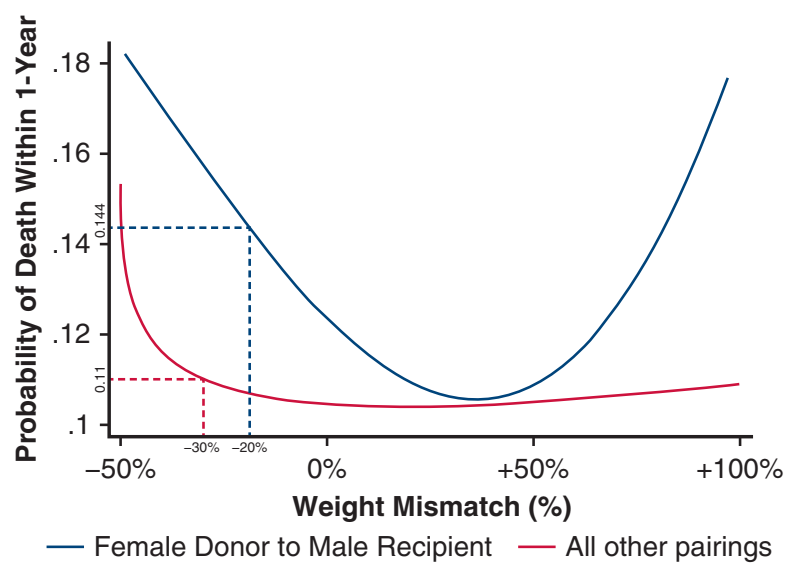

FIGURE 4. Fractional polynomial regression models demonstrating the probability of death within 1 year of transplantation by weight mismatch. Blue indicates female donor to male recipient $(\mathrm{n}=6071)$, and red indicates all sex matching results except female donor to male recipient $(n=32,669)$. These curves demonstrate outcomes of current International Society of Heart and Lung Transplantation guidelines, which state that "the use of hearts from donors whose body weight is no greater than $30 \%$ below that of the recipient is uniformly safe. Use of a female donor whose weight is more than $20 \%$ lower than that of a male recipient should be viewed with caution." Guidelines on both graphs delineate the projected 1-year mortality risk associated with each of these statements, demonstrating the complexity of and discrepancy in risk profiles. match $<0 \%$ ) had a higher rate of female donor to male recipient HTx, and as RVM mismatch became more positive, the proportion of female donor to male recipient HTX decreased and that of male donor to female recipient HTx increased. No significant difference was seen in the proportion of recipients with an LVAD.

Interestingly, although sex matching with female donors to male recipients has been previously reported as a risk factor, ${ }^{3}$ the undersized group (RVM match $<0 \%$ ) had a younger recipient population yet still demonstrated a higher 1 -year mortality rate. Wever-Pinzon and colleagues ${ }^{15}$ reported a significant survival difference across recipient age groups, with larger and significant differences for recipients in their 60s and 70s but no significant difference for those in their $40 \mathrm{~s}$ and $50 \mathrm{~s} .{ }^{15}$ The mean recipient age in our study was in the 40 s to $50 \mathrm{~s}$; likely RVM mismatch played a greater role than age in the decreased survival seen in RVM undersizing.

In our analysis, RVM constituted approximately $13 \%$ to $15 \%$ of TVM (13.2\% in donors, $15.5 \%$ in recipients). Two points should be made concerning this finding. First, the RVM proportion in TVM was small. Second, donor TVM and RVM were higher in the donors than in the recipients. This difference could be related to age, with younger donors having greater RVM and TVM compared with older donors.

In a 2015 American Society of Transplantation Conference online survey of 47 transplantation centers, in the donor to recipient ratio, the greatest importance was assigned to height by $57 \%$ of the respondents and to weight by $43 \%$. For a female donor heart and male recipient, $46 \%$ of respondents would oversize the donor; however, impressively, $48 \%$ believed that oversizing would not be necessary, and $6 \%$ would accept an undersized heart. ${ }^{19}$ Our analysis of the current ISHLT guideline recommendations showed that female donor to male recipient HTx was associated with higher mortality than other sex matches in any weight match. The $<-20 \%$ 
Right Ventricular Mass Undersizing is Associated with Increased One-Year Mortality

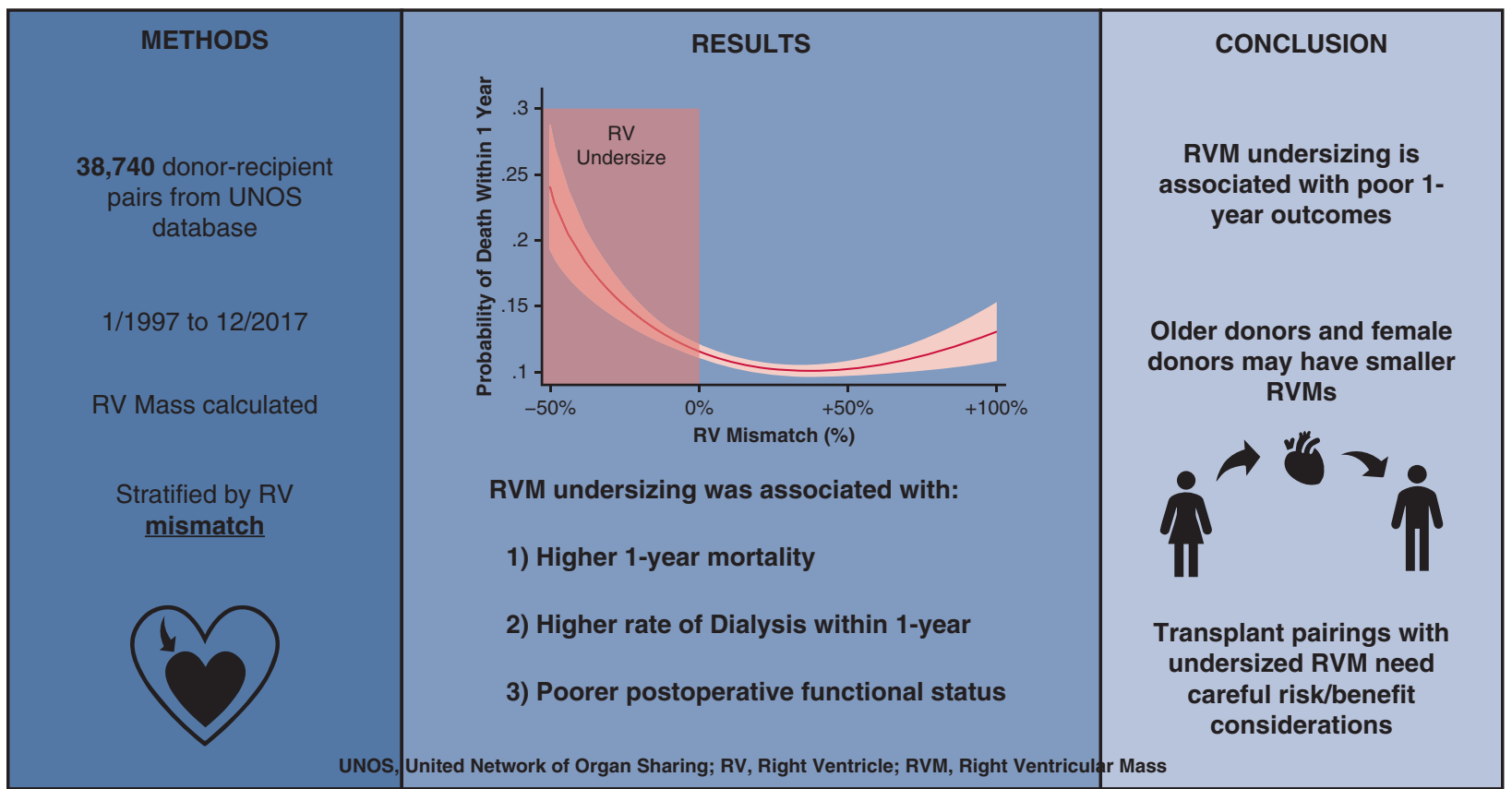

FIGURE 5. Outline of the methods and primary findings of our study. A UNOS database analysis of $>38,000$ donor-recipient pairs between 1997 and 2017 was performed. Fractional polynomial regression analysis identified an association between right ventricular undersizing and a higher risk of 1-year mortality. Other associations include a higher rate of dialysis and poorer postoperative functional status.

TVM match in this group was associated with $>13.8 \%$ 1 -year mortality. We even saw elevated risk when weight matching was $>-20 \%$. Female donor to male recipient pairing may need to be viewed with further caution and risk-benefit consideration, even more so than weight matching, given that an even more conservative matching cutoff of $>-20 \%$ was still associated with worse outcomes. These findings are consistent

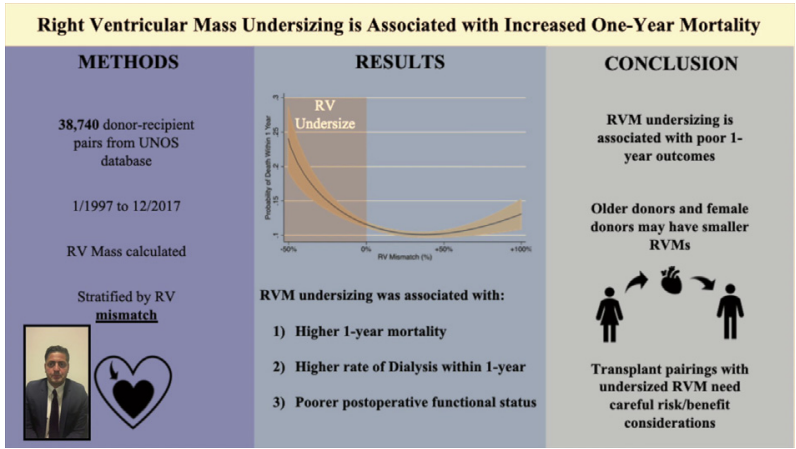

VIDEO 1. Dr Critsinelis describing the primary findings of this study, emphasizing the study's importance and implications for current donor heart selection practices. Video available at: https://www.jtcvs.org/ article/S0022-5223(20)33318-3/fulltext. with a previous study by Khush and colleagues exploring this topic. ${ }^{3}$ On the other hand, the nonfemale donor to male recipient population had a low risk in guideline-recommended weight match.

\section{RVM Calculator}

Using data from the UNOS database and the analysis from this manuscript, we developed a free access RVM donor-recipient matching calculator website (rvmcalc. com). Users can instantaneously calculate estimated RVM, TVM, and mismatch by entering donor and recipient age, sex, height, and weight. Figure $3, A$ and $C$, provides a reference. The calculator is not a substitute for clinical judgment but instead should be used in conjunction with established guidelines and clinician experience. The site can be accessed from computer, tablet, and smart phone.

\section{Limitations}

We recognize that our study has some limitations. This was a retrospective analysis and thus subject to the limitations inherent to this study type. We used the UNOS database, a large multi-institutional dataset, which may lack the granularity of single-institutional data, such as detailed postoperative outcomes. The data and results reflect 20 years of HTx data in the United States, which may incorporate the effects of multiple changes in allocation, distribution, 
ISHLT guidelines, strategies used by transplantation centers that come with the use of mechanical circulatory support, and/or donor-recipient sex matching recommendations during this period. Furthermore, there remain some biases and judgment in recipient listing, allocation policy, and donor acceptance. There are also limitations in the equations used to estimate ventricular mass. Using an RVM matching calculation places less importance on LV factors compared with TVM calculation. Finally, results from RVM matching may overemphasize the effects of age and sex matching, particularly in cases with large age gaps, such as older donors with younger recipients.

Future studies are needed to further explore the RVM matching relationship, particularly the increased mortality risk seen with gross RVM oversizing seen in our analysis. In-depth analyses should also aim to identify patients who are at low risk and high risk for morbidity and mortality associated with RVM undersizing.

\section{CONCLUSIONS}

$\mathrm{RV}$ performance is critical after $\mathrm{HTx}$, and assessing RVM might be useful in predicting outcomes. We analyzed donor RVM and its effect on HTx results using the UNOS registry. Elderly and female donors have lower absolute RVM. We found that undersizing RVM was associated with poorer outcomes. RVM-undersized HTx requires careful recipient risk-benefit consideration under clinical experience (Video 1 and Figure 5).

\section{Webcast}

You can watch a Webcast of this AATS meeting presentation by going to: https://aats.blob.core.windows.net/media/ 20AM/Presentations/Right $\% 20$ Ventricular $\% 20$ Mass $\%$ 20Undersizing \%20i.mp4.

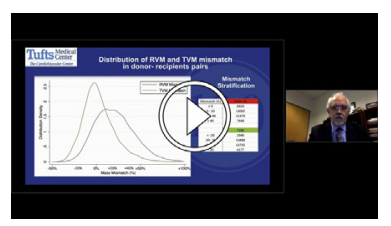

\section{Conflict of Interest Statement}

The authors reported no conflicts of interest.

The Journal policy requires editors and reviewers to disclose conflicts of interest and to decline handling or reviewing manuscripts for which they may have a conflict of interest. The editors and reviewers of this article have no conflicts of interest.

\section{References}

1. Costanzo MR, Dipchand A, Starling R, Anderson A, Chan M, Desai S, et al. The International Society of Heart and Lung Transplantation guidelines for the care of heart transplant recipients. J Heart Lung Transplant. 2010;29:914-56.
2. Patel ND, Weiss ES, Nwakanma LU, Russell SD, Baumgartner WA, Shah AS, et al. Impact of donor-to-recipient weight ratio on survival after heart transplantation: analysis of the United Network for Organ Sharing database. Circulation. 2008;118(14 Suppl):S83-8.

3. Khush KK, Kubo JT, Desai M. Influence of donor and recipient sex mismatch on heart transplant outcomes: analysis of the International Society for Heart and Lung Transplantation registry. J Heart Lung Transplant. 2012; 31:459-66.

4. Bluemke DA, Kronmal RA, Lima JAC, Liu K, Olson J, Burke GL, et al. The relationship of left ventricular mass and geometry to incident cardiovascular events: the MESA (Multi-Ethnic Study of Atherosclerosis) study. J Am Coll Cardiol. 2008;52:2148-55.

5. Kawut SM, Lima JAC, Barr RG, Chahal H, Jain A, Tandri H, et al. Sex and race differences in right ventricular structure and function: the multi-ethnic study of atherosclerosis-right ventricle study. Circulation. 2011;123:2542-51.

6. Gong TA, Joseph SM, Lima B, Gonzalez-Stawinski GV, Jamil AK, Felius J, et al. Donor predicted heart mass as predictor of primary graft dysfunction. $J$ Heart Lung Transplant. 2018;37:826-35.

7. Kransdorf EP, Kittleson MM, Benck LR, Patel JK, Chung JS, Esmailian F, et al Predicted heart mass is the optimal metric for size match in heart transplantation. J Heart Lung Transplant. 2019;38:156-65.

8. Bergenfeldt H, Lund LH, Stehlik J, Andersson B, Höglund P, Nilsson J. Time dependent prognostic effects of recipient and donor age in adult heart transplantation. J Heart Lung Transplant. 2019;38:174-83.

9. Reed RM, Netzer G, Hunsicker L, Mitchell BD, Rajagopal K, Scharf S, et al. Cardiac size and sex-matching in heart transplantation: size matters in matters of sex and the heart. JACC Heart Fail. 2014;2:73-83.

10. Brumback LC, Kronmal R, Heckbert SR, Ni H, Hundley WG, Lima JA, et al Body size adjustments for left ventricular mass by cardiovascular magnetic resonance and their impact on left ventricular hypertrophy classification. Int J Cardiovasc Imaging. 2010;26:459-68.

11. Chirinos JA, Segers P, De Buyzere ML, Kronmal RA, Raja MW, De Bacquer D, et al. Left ventricular mass: allometric scaling, normative values, effect of obesity, and prognostic performance. Hypertension. 2010;56:91-8.

12. Armstrong AC, Gidding S, Gjesdal O, Wu C, Bluemke DA, Lima JAC. LV mass assessed by echocardiography and CMR, cardiovascular outcomes and medical practice. J Am Coll Cardiol. 2012;5:837-48.

13. Chambers DC, Cherikh WS, Harhay MO, Hayes D Jr, Hsich E, Khush KK, et al. The international thoracic organ transplant registry of the International Society for Heart and Lung Transplantation: thirty-sixth adult lung and heart-lung transplantation report-2019; focus theme: donor and recipient size match. J Heart Lung Transplant. 2019;38:1042-55.

14. Russo MJ, Hong KN, Davies RR, Chen JM, Mancini DM, Oz MC, et al. The ef fect of body mass index on survival following heart transplantation: do outcomes support consensus guidelines? Ann Surg. 2010;251:144-52.

15. Wever-Pinzon O, Edwards LB, Taylor DO, Kfoury AG, Drakos SG, Selzman CH, et al. Association of recipient age and causes of heart transplant mortality: implications for personalization of post-transplant management-an analysis of the International Society for Heart and Lung Transplantation registry. J Heart Lung Transplant. 2017;36:407-17.

16. Weber DJ, Wang IW, Gracon ASA, Hellman YM, Hormuth DA, Wozniak TC et al. Impact of donor age on survival after heart transplantation: an analysis of the United Network for Organ Sharing (UNOS) registry. J Card Surg. 2014;29: 723-8.

17. Jawitz OK, Raman V, Klapper J, Hartwig M, Patel CB, Milano C. Donor and recipient age matching in heart transplantation: analysis of the UNOS registry Transpl Int. 2019;32:1194-202.

18. Schumer EM, Black MC, Rogers MP, Trivedi JR, Birks EJ, Lenneman AJ, et al Donor oversizing results in improved survival in patients with left ventricular assist device. ASAIO J. 2016;62:571-7.

19. Kobashigawa J, Khush K, Colvin M, Acker M, Van Bakel A, Eisen H, et al. Report from the American Society of Transplantation conference on donor heart selection in adult cardiac transplantation in the United States. Am J Transplant. 2017; 17:2559-66.

Key Words: heart transplantation, United Network for Organ Sharing registry, predicted heart mass, right ventricular mass, total ventricular mass, survival, donor and recipient heart size, donor-recipient heart match, undersizing 


\section{Discussion}

\section{Presenter: Dr Gregory S. Couper}

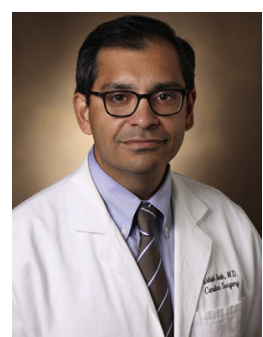

Dr Ashish S. Shah (Nashville, Tenn). I

would like to thank the Association for the opportunity to discuss this paper and the authors for providing the slides in advance. Despite over 50 years of clinical heart transplantation, the decision to use a heart for transplant rests in the hands of the implanting surgeon, usually late at night, often in the context of multiple forces, including hospital-based resources, regulatory pressures, and fatigue.

The criteria for what is considered an acceptable donor have remained essentially unchanged over the decades and vary from center to center. I think we all worry about using small female hearts, which remain underutilized, certainly in the United States. Over the last several years, however, we have had an emerging and more nuanced view of what constitutes an acceptable donor. Because we're incorporating large datasets and quantifying risk relative to the recipient (not just the donor in isolation), that has helped teams make better decisions. Even more recently, we've been introduced to the idea of myocardial mass between the donors and the recipients. The recent paper from the Cedars-Sinai group examining predicted heart mass and postoperative outcomes has certainly influenced our thinking at Vanderbilt, and I sincerely believe the authors of this study have added another novel dimension in considering right ventricular mass.

You mentioned this earlier, but while 1-year survival is significantly different, the absolute difference is small at $2 \%$. In your multivariable model, your Cox proportional hazards model, it is statistically significant, and you mentioned it—do you think this is clinically meaningful? And the second part of this question is: Have you considered exploring other endpoints, such as the need for postoperative mechanical support, renal failure, and functional status?

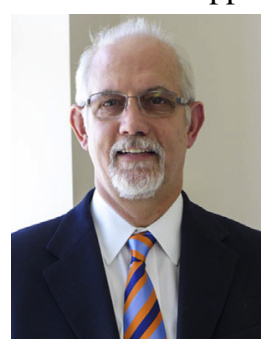

Dr Gregory S. Couper (Boston, Mass). We did look at the outcomes other than survival, and in that lowest $\mathrm{RV}$-matched group, there was a little bit more graft failure. And of course, the definitions of PGD evolved over the course of the study, and what people inputted as graft failure over our 21 years of database entry could vary.

There was a little bit more rejection, a little bit more renal failure, a little bit more postop dialysis, a little bit more multiorgan failure. We can't pin down what exactly is creating the small difference in mortality. I've always believed that all these things likely roll back to the performance of the allograft right off the bat. It is a relatively small difference, but one of the things we'd like to explore further is whether in this population we can develop the subpopulation in which we really find that this difference is dramatic - where it's not diluted by the vast numbers of patients. The preoperative hemodynamics-pulmonary artery pressure and all those things-were the same in all groups. So that's not really the answer. But it's something we have to explore further by really taking our group of interest and taking the signal and seeing if we can somehow amplify it.

Dr Shah. Other studies comparing donor-recipient matching have found an important relationship in preexisting pulmonary hypertension. So maybe restate your conclusion. Did you consider preoperative pulmonary hypertension in this model? Is there any interaction between the undersized donors and preoperative pulmonary hypertension?

Dr Couper. In that group of the lowest RV-mismatched group of less than $0 \%$, it's a broad range. It covers a broad range of the LV-mismatched patients below $0 \%$. We could not identify, on the whole, that there was a significant difference in pulmonary artery pressure. Pulmonary vascular resistance, I don't recollect it necessarily being reported frequently or well.

Dr Shah. Fair enough. It's certainly something to think about it. We've looked at some of this again with UNOS data and it did seem that undersized donors in patients with pulmonary hypertension, you know, plus or minus an LVAD, you start adding some of these variables together and it may be that your RV mass calculation might be a nice added variable to put in the mix, particularly if you think about this as a risk factor among others. My third question is: Why do you think the oversizing had a mortality to it?

Dr Couper. When you begin to express these curves in that parabolic sense, it really becomes obvious that oversizing seems to be almost as big a problem. I don't know the answer to that. My medical colleague Amanda Vest spends most of her clinical and basic research looking into nutritional factors, especially morbid obesity and its impact. These would be obese donors (or more obese donors, presumably) and more male donors at that end of the spectrum. Dr Vest is convinced that the morphologic and phenotypic changes of the obese heart rapidly reverse after transplantation. Certainly within a year of bariatric surgery, there are marked changes toward normalization. I don't know that we know that after transplantation. I think all of us may have had a hard time fitting a really big heart into a space that won't take it, but I don't really think that that's the answer.

Dr Shah. All these calculations are based on normal hearts. So if we're able to use calculations based on abnormal hearts (that is, recipients who have restrictive myopathies, hypertrophy or dilated myopathies), can you speculate how relevant these normal heart calculations are to our recipients? 
Dr Couper. I would agree with you there. These equations were developed to describe the general population, and they probably describe the donor population pretty well, and then we've obviously skewed our selection of donors. These subgroups of patients with smaller-volume hearts, but more dense mass are fascinating, and it would take quite a bit more work, and I'm not sure that we'll have the statistical power there to really make firm statements. But it's an interesting idea.

Dr Shah. Well, thank you for that presentation. I just have one comment. I think we do need to start moving away from survival as the as the only endpoint, particularly thinking about future allocation systems. There really are other important functional endpoints like exercise capacity, long-term survival, quality of life that may really affect how we utilize hearts and how we utilize these calculations. Sure, we can get them through the operation-but have we really just transplanted heart failure at a year? So thanks again for the opportunity.

Dr Couper. Thank you.

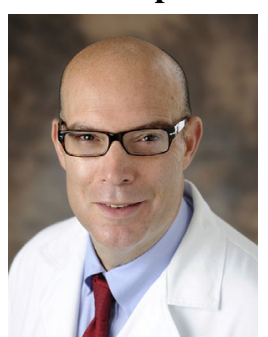

Dr Scott C. Silvestry (Orlando, Fla). First, congratulations. I think this study is a phenomenal analysis of an important aspect of transplantation. Eberlein and coauthors reported the first predicted heart mass data in JACC Heart Failure in 2014, and we have been using predictive heart mass to guide clinical decisions. Your analysis takes this thinking to another level. If you drill down on your right ventricular mismatched population and look at preop PVR, you will find that the real survival disadvantage occurs in PVRs greater than 2.5.

We have single-center data looking at that, but it's not enough to see-but I think if you rerun your data by mismatch in the pulmonary vascular resistance, there are certain susceptible populations, and I believe this is a sort of a hint of it. I'm glad you're putting the RV calculator out there. My question to you on this data is: How is this changing the decisions that you're making in these patients today moving forward?

Dr Couper. I agree with you and Dr Shah that basically, the recipients with elevated pulmonary vascular resistance are probably the fertile area to study further. They're probably the ones at risk of RV mass undersizing. This dataset has not yet really impacted how we look at donor selection. I think a lot of these are attempts to quantify what our surgical intuition has been for decades and how we pick things. I would say that using the total cardiac mass calculator, I am surprised at times that there's a better match, especially younger, female, pretty heavy donors. We've taken a whole lot more of those in recent years than I probably would have dreamed of maybe 5 or 10 years ago.

So I think that by quantifying these things, it brings us to a realization that it's okay. The other thing I would like to just say about the calculators and these parabolic curves is that there are no breakpoints in any of these curves. So there are no absolute thresholds or cutoffs. I think it's all going to be a relative risk-benefit ratio thought process, you know at 2 a.m.

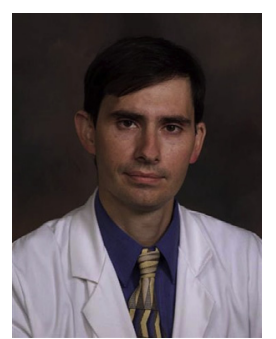

Dr Carmelo A. Milano (Durham, $N C$ ). I think the other thing that makes this discussion more complicated is that our conventional cold static storage does induce some degree of injury to the right ventricle. And usually that's not particularly significant, but I think in many instances it is significant, and it occurs in an unpredictable manner. So I think continuing to look toward better preservation strategies is a very important topic for our field, and it may affect the way we select organs and what we can get away with and what we can't get away with. Again, thank you for that interesting presentation. Thank you for the discussion around it. 


\section{UNOS Database:}

Primary adult heart transplants from January 1997-December 2017

$$
\mathrm{N}=49,819
$$

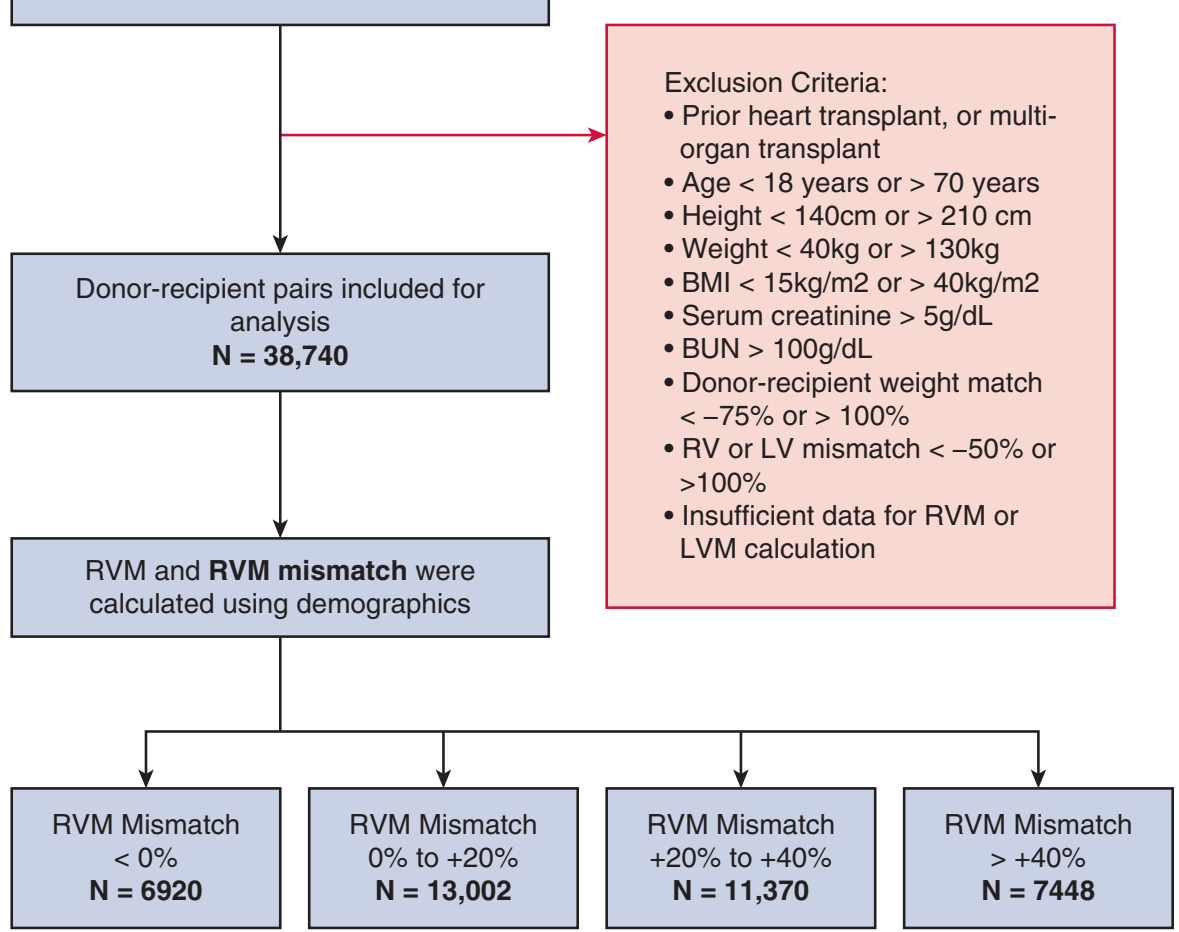

FIGURE E1. PRISMA flow diagram demonstrating inclusion and exclusion criteria for our study. UNOS, United Network for Organ Sharing; BMI, body mass index; $B U N$, blood urea nitrogen; $R V$, right ventricle; $L V$, left ventricle; $R V M$, right ventricular mass; $L V M$, left ventricular mass.

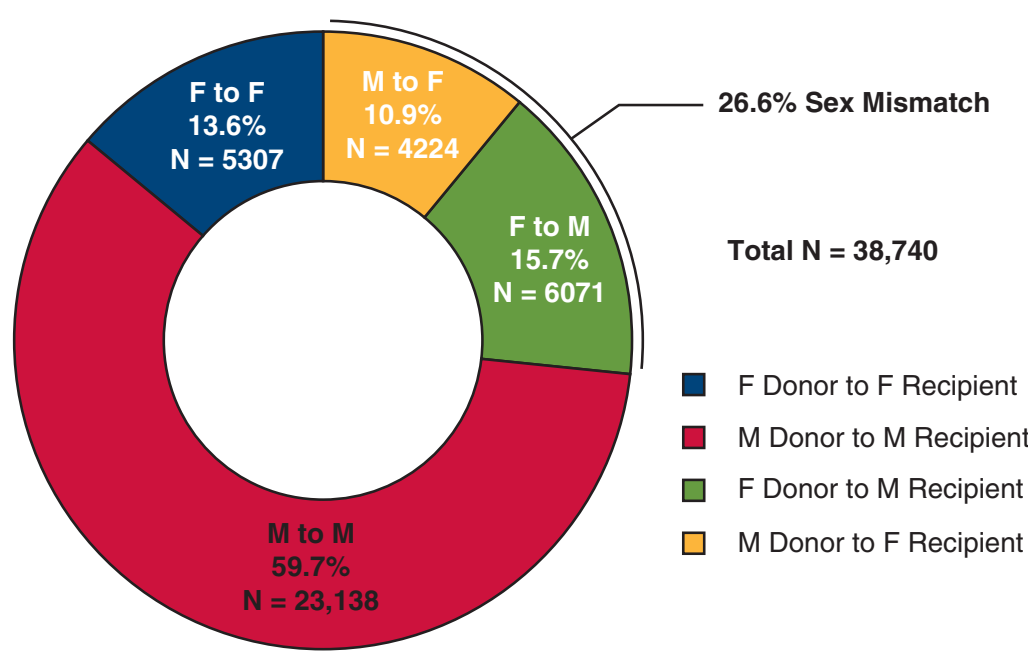

FIGURE E2. Distribution of sex in donor-recipient pairs in heart transplantations included in our study. There was a $26.6 \%$ rate of sex mismatch, and a $15.7 \%$ rate of female $(F)$ donor to male $(M)$ recipient pairings. 


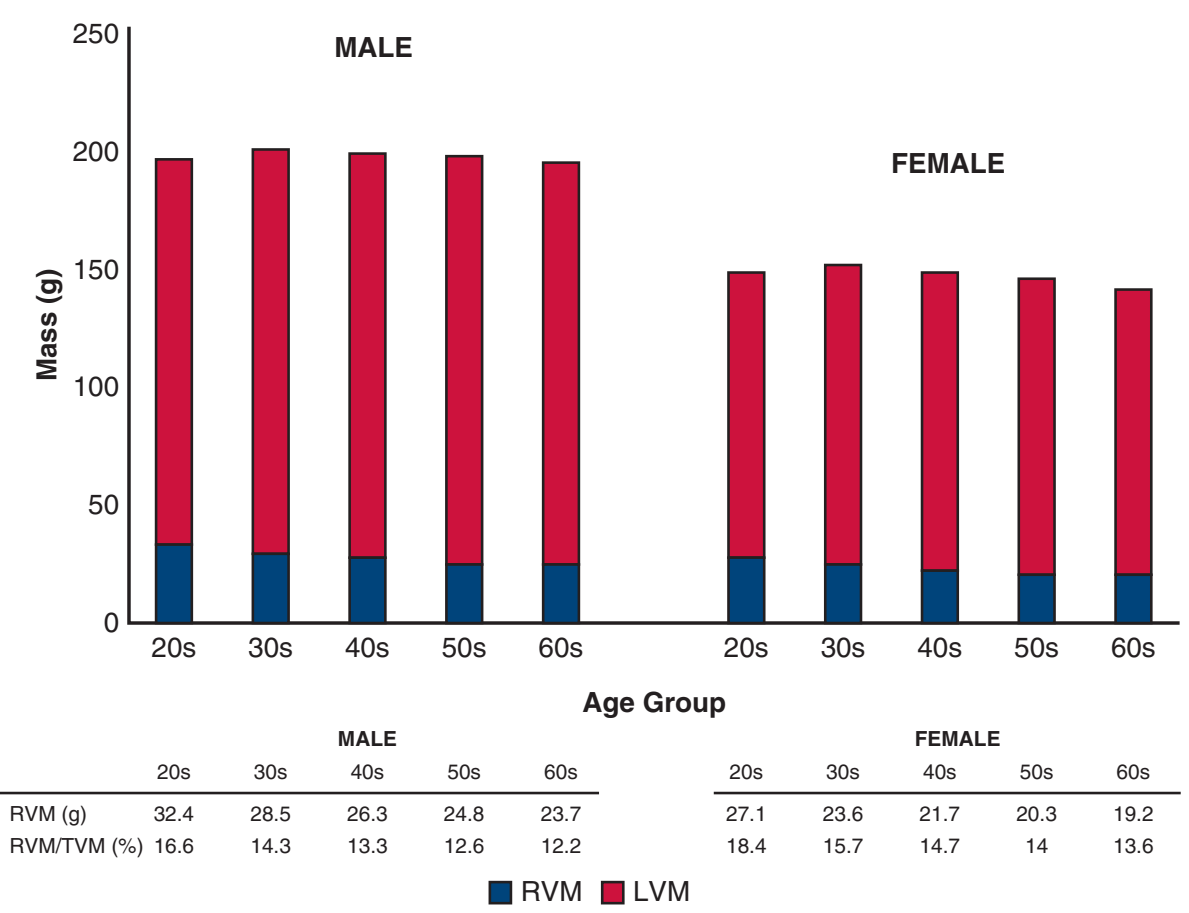

FIGURE E3. Average left ventricular mass (LVM; blue), right ventricular mass (RVM; red), and total ventricular mass (TVM; stacked bar consisting of both red and blue) for heart transplant donor hearts included in our study. Values are stratified by age group and sex. Male donors had greater RVM and LVM compared with female donors, and RVM showed a gradual decrease with increasing donor age. The groups with smallest RVM were female donors age $\geq 60$ years. 


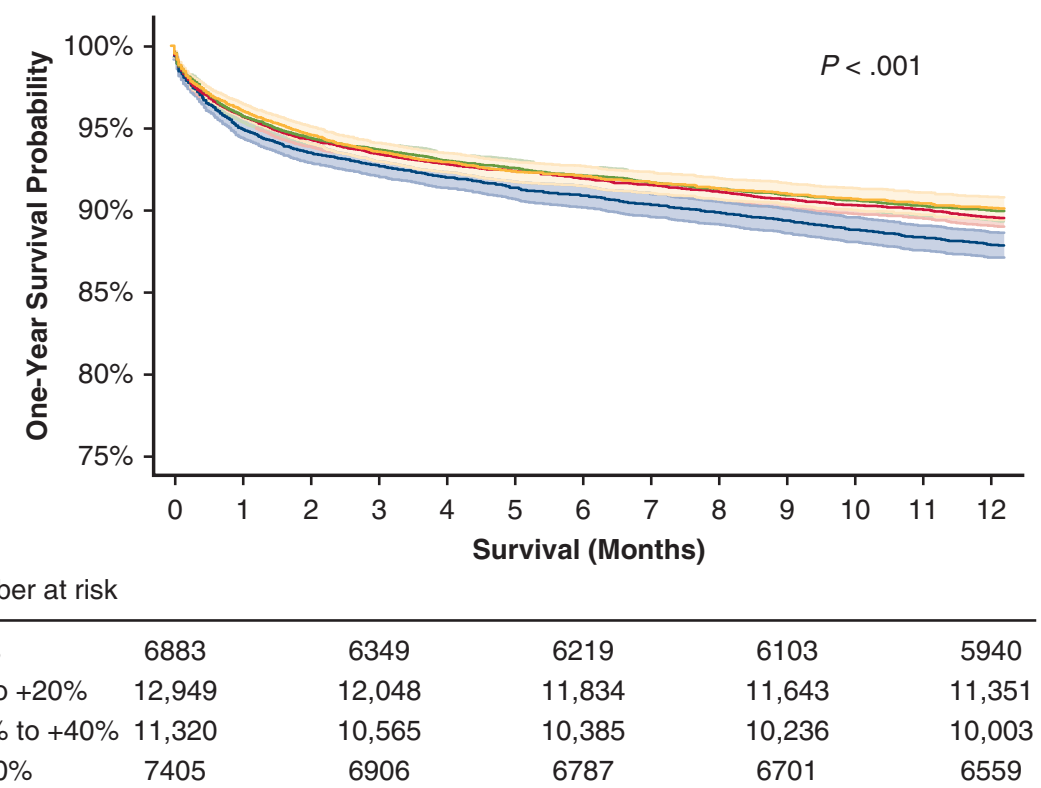

A

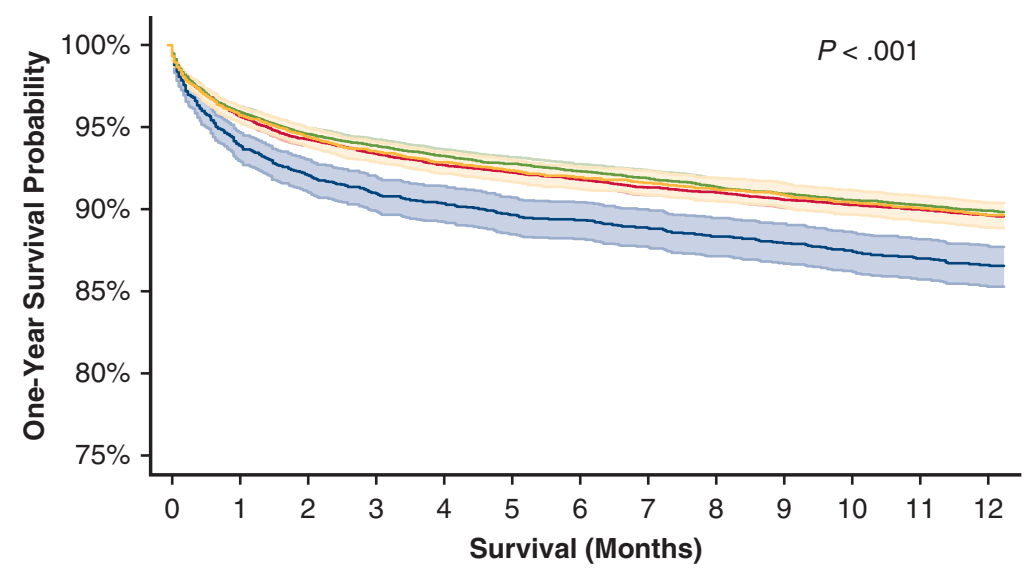

Number at risk

\begin{tabular}{cccccc}
\hline$-<-20 \%$ & 2924 & 2646 & 2596 & 2554 & 2483 \\
$--20 \%$ to $0 \%$ & 15,812 & 14,711 & 14,453 & 14,227 & 13,901 \\
$-0 \%$ to $+20 \%$ & 13,685 & 12,799 & 12,571 & 12,368 & 12,081 \\
$->+20 \%$ & 6136 & 5712 & 5605 & 5534 & 5388
\end{tabular}

B

FIGURE E4. Similar to Figure 2, this figure shows Kaplan-Meier curves demonstrating 1-year survival stratified by right ventricular mass (RVM) mismatch group (A) and total ventricular mass (TVM) mismatch group (B). Corresponding $95 \%$ confidence interval bands are demarcated by shading. 